TJVR 2021; 5 (2): 99-104

Turkish Journal of Veterinary Research

https://dergipark.org.tr/tr/pub/tjvr

e-ISSN: 2602-3695

\title{
The effect Chia seeds (Salvia hispanica L.) on gastrointestinal echogenicity in cats
}

\author{
Burcu Gökdemirel Kılıç1® Zeynep Bozkan'10 \\ ${ }^{1}$ Department of Surgery, Faculty of Veterinary Medicine, Aydin Adnan Menderes University, Aydın, Turkey
}

Correspondence: Zeynep Bozkan (zbozkan@adu.edu.tr)

Received: 07.10.2021

Accepted: 20.10 .2021

\begin{abstract}
Objective: It was aimed to investigate whether chia seeds provide an advantage during ultrasonographic imaging in cats when added to food by means of its high water-holding capacity.
\end{abstract}

Materials and Methods: Ten healthy cats were ultrasonographically examined following 12 - hour fasting period and after feeding with 3 different food types at 1-week intervals. Thus, 4 study group were formed as follows; FST; fasting 12- hour period, WF; feeding with wet cat food following 12- hour fasting period DWF; feeding with $1 / 2$ of same wet food diluted with the same volume drinking water, CWF; feeding with $1 / 2$ of same wet food mixed with $36 \mathrm{ml}$ swollen chia seeds obtained by holding 2 hours $3 \mathrm{ml}$ chia seeds in 33 $\mathrm{ml}$ drinking water. Cats were evaluated ultrasonographically immediately after feeding, and at the 30th minutes and 60th minutes.

Results: Especially in terms of thickness, gastric wall evaluation was easier after food intake. In the CWF, the stomach was fully imaged on the frame at all times. The gastrointestinal tract content moved faster when cats were fed DWF or CWF; In the examination immediately after feeding the contents were in duodenum and seen in the jejunum and ileum in the 30- and 60-minutes' examinations. CWF and DWF revealed more heterogeneous images then WF and also CWF provided better visualization for the intestinal canal wall layer.

Conclusion: Ultrasonographic examination after feeding with chia seed added formula can be used as a complementary method following the fasting examination.

Keywords: Cat, Chia seed, Echogenicity, Food, Gastrointestinal, Ultrasonography

\section{INTRODUCTION}

Ultrasonography is an imaging method based on the principle of sending high-frequency sound waves to the area of the body to be examined and the reflection of these waves in different tissues (Burk and Feeney, 2003). Gastrointestinal tract ultrasonography is often performed for cases of acute abdominal pain, vomiting, diarrhea, melena, intestinal wall thickening, and also it is used for determination of dilatation and obstruction of the intestines, presence of palpable masses, investigation of mesenteryomentum and peritoneal masses (Agthe, 2009).
Most sources suggest 12- hour fasting before the ultrasonographic examination, and also state that giving water to the patient before the examination provides a better assessment of the stomach wall (Penninck, 2008; Mattoon, 2009). It is even recommended to remove the intraluminal gas and fill the stomach with water by applying gastric catheter in humans and animals (Machi et al., 1986; Penninck et al., 1989), because the intraluminal gas causes frequently artefacts such as reverberation, comet-tail and acoustic shadow (Burk and Feeney, 2003; Peiretti and Meineri, 2008). 
There is no much information about how the composition of the content remaining in the digestive tract affects the echogenicity during ultrasonographic imaging (Gaschen et al., 2016). Normal intestinal mucosa can be considered almost as anechoic, and some recent studies in dogs suggest that food intake and food quality will increase digestive tract echogenicity (Pollard et al., 2013; Gaschen et al., 2016).

Chia seeds (Salvia hispanica L.) is a unique food substance is known as hydrophilic, absorbing up to 12 times their weight in liquid when soaked and forms a gel layer around it (Muñoz et al., 2012). Also, it is used in many studies for human and animal nutrition due to its nutritional and therapeutic properties (Ayerza et al., 2002; Peiretti and Meineri, 2008; Ullah et al., 2016). In addition, since it does not have a noticeable taste and smell, it is easily consumed by the animals when mixed in to the food (Ullah et al., 2016). It was hypothesized that food intake containing swollen Chia seeds before ultrasonographic examination will positively affect the echogenicity in ultrasonographic examination due to the fact that sound waves propagation is much better in liquid and gel environments.

\section{MATERIALS and METHODS}

\section{Animals}

Study material was 10 healthy cats from different breed, gender, age and weight brought to our clinics (Table 1).

Table 1. Signalmen of the cats that constitutes study material

\begin{tabular}{llcc}
\hline No & Breed & $\begin{array}{c}\text { Age } \\
\text { (year) }\end{array}$ & Gender \\
\hline 1 & Mix & 6 & Male \\
2 & Chinchilla & 11 & Female \\
3 & British Short Hair & 3 & Male \\
4 & Persian-Himalayan Mix & 1 & Female \\
5 & Persian-Himalayan Mix & 1 & Male \\
6 & Mixed & 1.5 & Female \\
7 & Mixed & 5 & Female \\
8 & Exotic Short Hair & 6.5 & Female \\
9 & British Short Hair & 5 & Female \\
10 & Mixed & 1.5 & Male \\
\hline
\end{tabular}

This study was approved by Aydin Adnan Menderes University Animal Experiments Local
Ethics Committee (on 30/01/2018 and numbered 2018/022). All the procedures were conducted in accordance with good practices for animal experimentation. The owners of all animals were informed about the study and their approval was obtained. Neither sedation or anesthetic agent was administered to any cat before or during the study, nor was force-fed.

\section{Experimental groups}

The same 10 cats were fed with 3 different methods at a week interval, following 12 -hour fasting period. Firstly, ultrasonographic examination were performed after fasting period and then repeated immediately after feeding and at the 30th and 60th minutes. Thus, 4 different assessment groups were generated.

FST: Fasting 12-hour period; WF: feeding with wet cat food (Proplan Gourmet Gold Ground Tuna 72 $\mathrm{ml}$ ) following 12-hour fasting period; DWF: feeding with $1 / 2$ of same wet food diluted with same volume drinking water; CWF: feeding with $1 / 2$ of same wet food mixed with $36 \mathrm{ml}$ swollen chia seeds obtained by holding 2 hours $3 \mathrm{ml}$ chia seeds in $33 \mathrm{ml}$ drinking water.

\section{Ultrasonographic Examinations}

The abdominal area to be examined was shaved and local ultrasound gel was applied, and all examinations were performed in dorsal recumbency B-Mode imaging method by using 8.0 $\mathrm{MHz}$ probe (Mylab 30-Esaote, Genova, Italy). A rubric was generated (Table 2) and data was scored according to this table. After evaluation of each group within itself, a single score was revealed for each group in the light of the proportionally weighted data.

\section{RESULTS}

The findings are summarized and presented in Table 2. The rugal folds of the stomach could be imaged totally in all FST cats. The stomach enlarged after food intake and rugal folds became invisible in all cats. However, total images of the stomach were taken at all times in the CWF, and at the 30th and 60th minutes in most cats in the DWF. Because of hyperechogenicity and artifacts caused by the stomach content the total image could not be taken at any time point in WF. The stomach lumen was anechoic in the FST and echogenicity was significantly increased in the all postprandial evaluations. This hyperechogenicity became homogeneous hypoechoic at 60th minute in CWF, but did not change in the other feeding protocols. 
Table 2. Pre- and postprandial ultrasound findings at 0,30 and 60 minutes

\begin{tabular}{|c|c|c|c|c|c|}
\hline Stomach & Min. & FST & WF & DWF & CWF \\
\hline \multirow{3}{*}{$\begin{array}{c}\text { Mucosal } \\
\text { Echogenity }\end{array}$} & 0 & 1 & 1 & 1 & 1 \\
\hline & 30 & & 2 & 2 & 2 \\
\hline & 60 & & 2 & 2 & 1 \\
\hline \multirow{3}{*}{$\begin{array}{l}\text { Luminal } \\
\text { Echogenity }\end{array}$} & 0 & $\mathrm{AE}$ & $\mathrm{HE}$ & $\mathrm{HE}$ & HT \\
\hline & 30 & & $\mathrm{HE}$ & HE & HT \\
\hline & 60 & & $\mathrm{HE}$ & HE & HE \\
\hline \multirow{3}{*}{$\begin{array}{l}\text { Viewing } \\
\text { the entire } \\
\text { stomach }\end{array}$} & 0 & + & - & - & + \\
\hline & 30 & & - & + & + \\
\hline & 60 & & - & + & + \\
\hline \multirow{3}{*}{$\begin{array}{l}\text { Rugal } \\
\text { Folds }\end{array}$} & 0 & + & - & - & - \\
\hline & 30 & & - & - & - \\
\hline & 60 & & - & - & - \\
\hline \multirow{3}{*}{ Pathologies } & 0 & - & - & - & - \\
\hline & 30 & & - & - & - \\
\hline & 60 & 0 & - & - & - \\
\hline \multicolumn{6}{|l|}{ Duedonum } \\
\hline \multirow{3}{*}{$\begin{array}{c}\text { Mucosal } \\
\text { Echogenity }\end{array}$} & 0 & 1 & 1 & 1 & 1 \\
\hline & 30 & & 2 & 2 & 1 \\
\hline & 60 & & 2 & 2 & 1 \\
\hline \multirow{3}{*}{$\begin{array}{c}\text { Luminal } \\
\text { Echogenity }\end{array}$} & 0 & $\mathrm{HO}$ & $\mathrm{HO}$ & HT & HT \\
\hline & 30 & & $\mathrm{HE}$ & HE & HT \\
\hline & 60 & & HE & HE & HT \\
\hline \multicolumn{6}{|l|}{ Jejenum } \\
\hline \multirow{3}{*}{$\begin{array}{c}\text { Mucosal } \\
\text { Echogenity }\end{array}$} & 0 & 1 & 1 & 1 & 1 \\
\hline & 30 & & 2 & 2 & 2 \\
\hline & 60 & & 2 & 1 & 1 \\
\hline \multirow{3}{*}{$\begin{array}{l}\text { Luminal } \\
\text { Echogenity }\end{array}$} & 0 & $\mathrm{HO}$ & $\mathrm{HO}$ & $\mathrm{HO}$ & $\mathrm{HO}$ \\
\hline & 30 & & HE & HT & HT \\
\hline & 60 & & $\mathrm{HE}$ & HT & HT \\
\hline \multicolumn{6}{|l|}{ Ileum } \\
\hline \multirow{3}{*}{$\begin{array}{c}\text { Mucosal } \\
\text { Echogenity }\end{array}$} & 0 & 1 & 1 & 1 & 1 \\
\hline & 30 & & 1 & 1 & 2 \\
\hline & 60 & & 1 & 1 & 1 \\
\hline \multirow{3}{*}{$\begin{array}{l}\text { Luminal } \\
\text { Echogenity }\end{array}$} & 0 & $\mathrm{HO}$ & HT & HT & HT \\
\hline & 30 & & HT & $\mathrm{HT}$ & HE \\
\hline & 60 & & HT & HT & HT \\
\hline
\end{tabular}

Mucosal Echogenity (Gaschen et al. 2016) = (0): Anechoic mucosa; (1): Small number of mucosal speckles present; (2): Large concentration of mucosal speckles present

Lumen= (A): Anechoic; (HO): Hypoechoic; (HT): Heterogeneous hyperechoic reflections in the hypoechoic field; (HE): Hyperechoic

Rugal Fold \& Pathology = (+): Present; (-): Absent

There was little mucosal hyperechogenicity in the all postprandial examinations at the first stage but then increased in all the feeding protocols.
Hyperechogenicity was decreased only in the CWF at the 60th minutes (Figure 1).

In the postprandial examination at the first, lumen of the duodenum was generally hypoechoic in the groups of FST and WF, and heterogeneous hypoechoic with hyperechoic areas in the DWF and CWF. Duodenum lumen became hyperechoic at the 30th and 60th minutes in WF and DWF, while it remained heterogeneous in CWF (Figure 2).

Hypoechogenicity of the jejunum lumen in fasted animals turned into hyperechogenicity at the 30th and 60th minutes postprandially in WF. In the other feeding protocols, the hyperechogenicity increased at the 30th minute and decreased in the 60th minute (Figure 3).

Hypoechoic ileum lumen in fasting became heterogeneous postprandially at the 0 , 30th and 60th minutes. Hyperechogenicity was dominant in most cats' ileum at the 30th minute only in the CWF (Figure 4).

\section{DISCUSSION}

In our study, considering the better propagation of sound waves in liquid and gel environments, the effect of food intake containing swollen Chia seeds (Salvia hispanica L.) on the ultrasonographic examination was investigated. Other advantage of Chia seeds in terms of its' usage in veterinary medicine is that it is easily consumed by animals when mixed into food since it has no noticeable taste and smell (Ullah et al., 2016). For the study, small particled food as possible was preferred to prevent artefacts that may arise from food particles, in the study.

Apart from this, it was also important that it is a product that cats love to eat to avoid force-feeding the cats. All cats included in the study ate voluntarily the food containing Chia seeds following a 12-hour fasting period.

Approaches and techniques may vary in ultrasonographic examination of the gastrointestinal system (Larson and Biller, 2009). The examination can be performed in standing, dorsal, right or left lateral recumbency for obtaining a more suitable acoustics in imaging (Penninck, 2008). However, imaging in the dorsal recumbency allows almost every part of the gastrointestinal system to be imaged (Larson and Biller, 2009). In our study, there was no problem obtaining the images of the targeted tissues in the dorsal recumbency. 


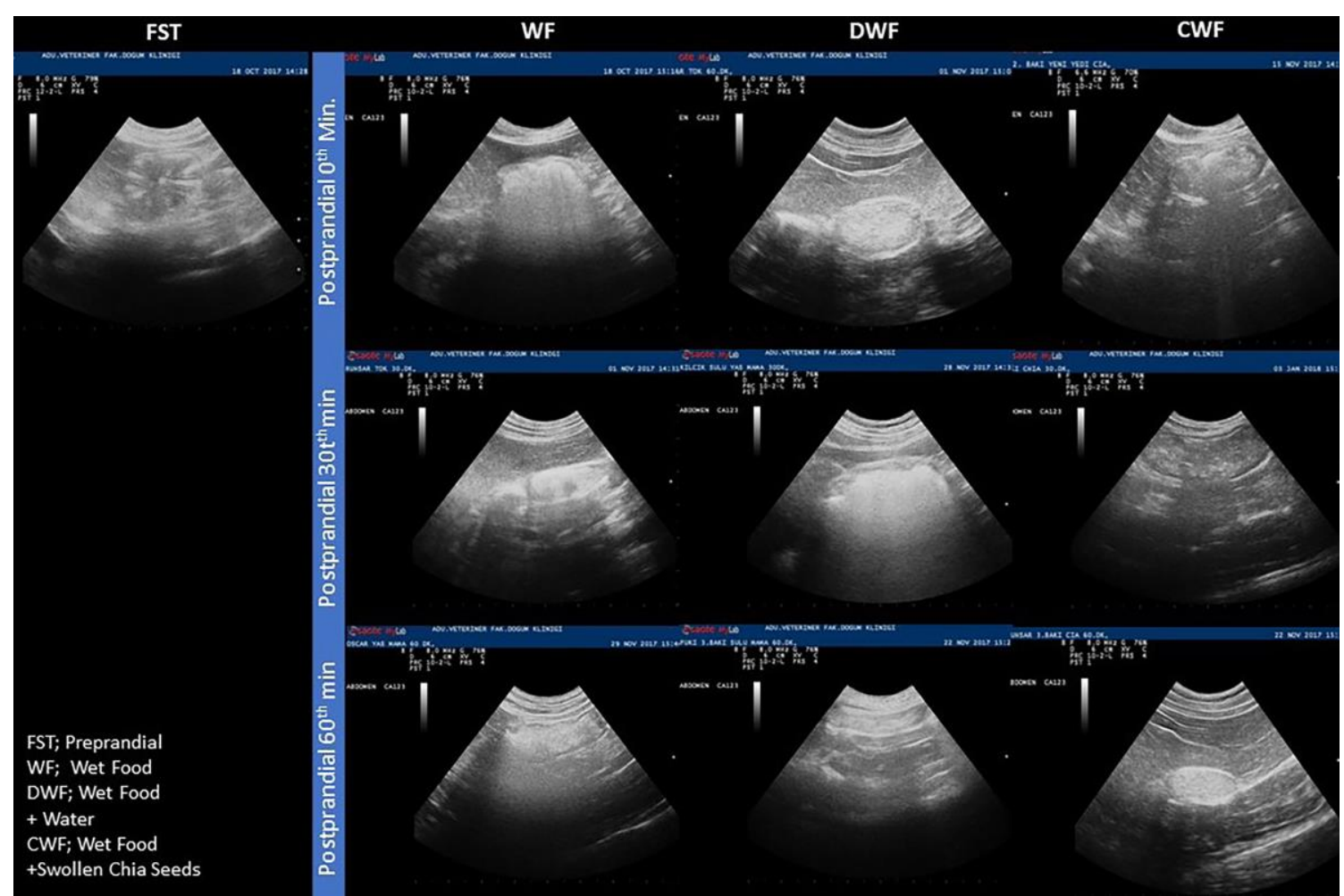

Figure 1. Ultrasonographic images of the stomach at pre- and postprandial 0, 30, 60 minutes

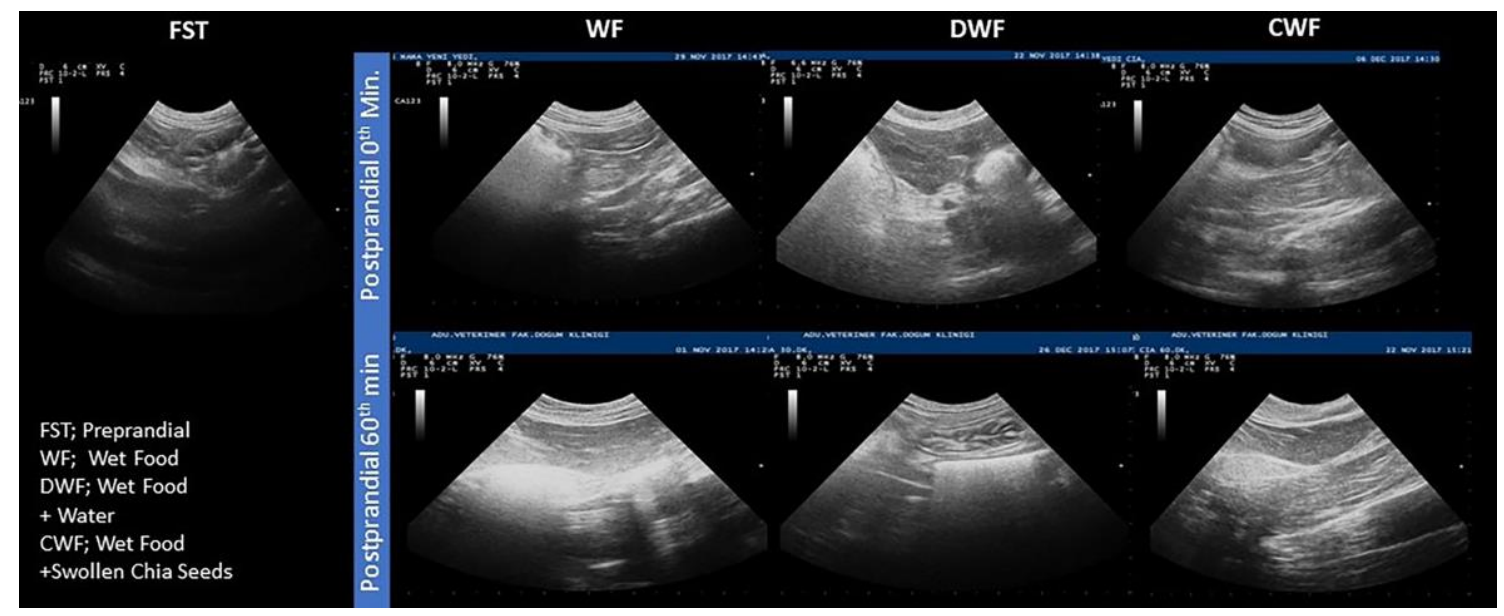

Figure 2. Ultrasonographic images of the duedonum at pre- and postprandial 0, 30, 60 minutes

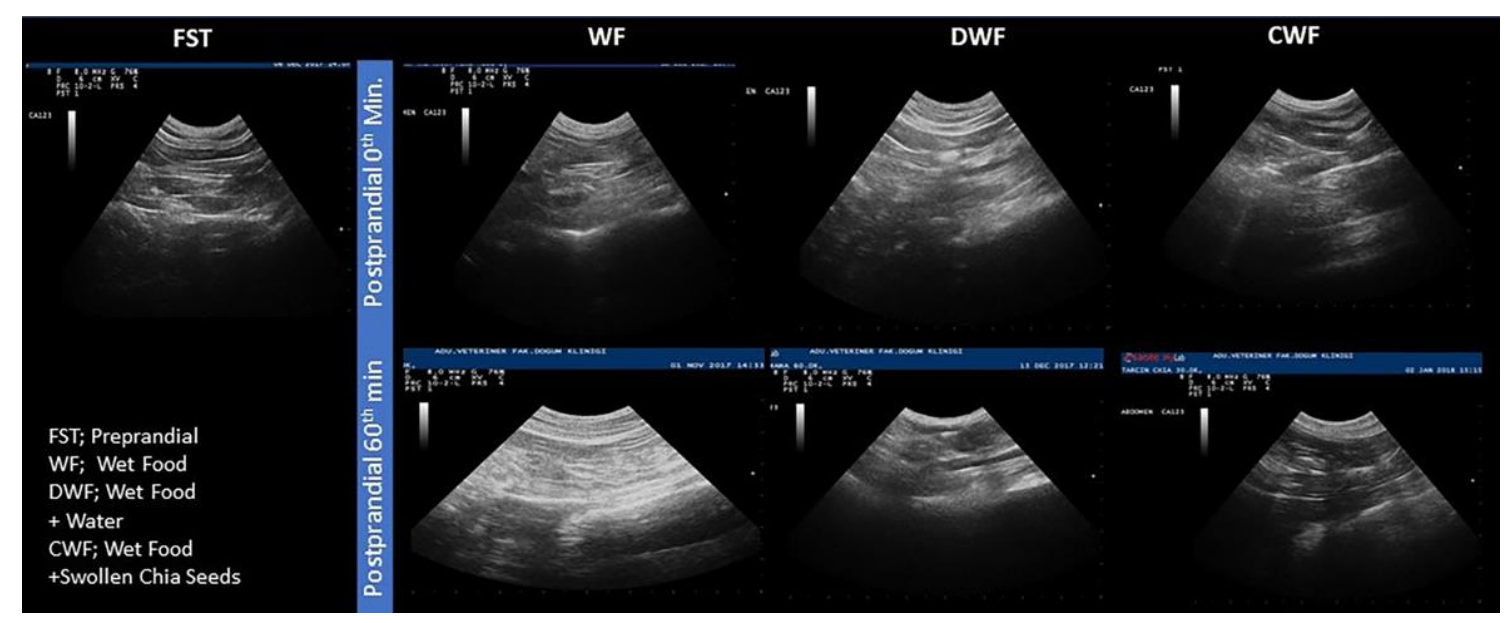

Figure 3. Ultrasonographic images of the jejenum at pre- and postprandial 0, 30, 60 minutes 


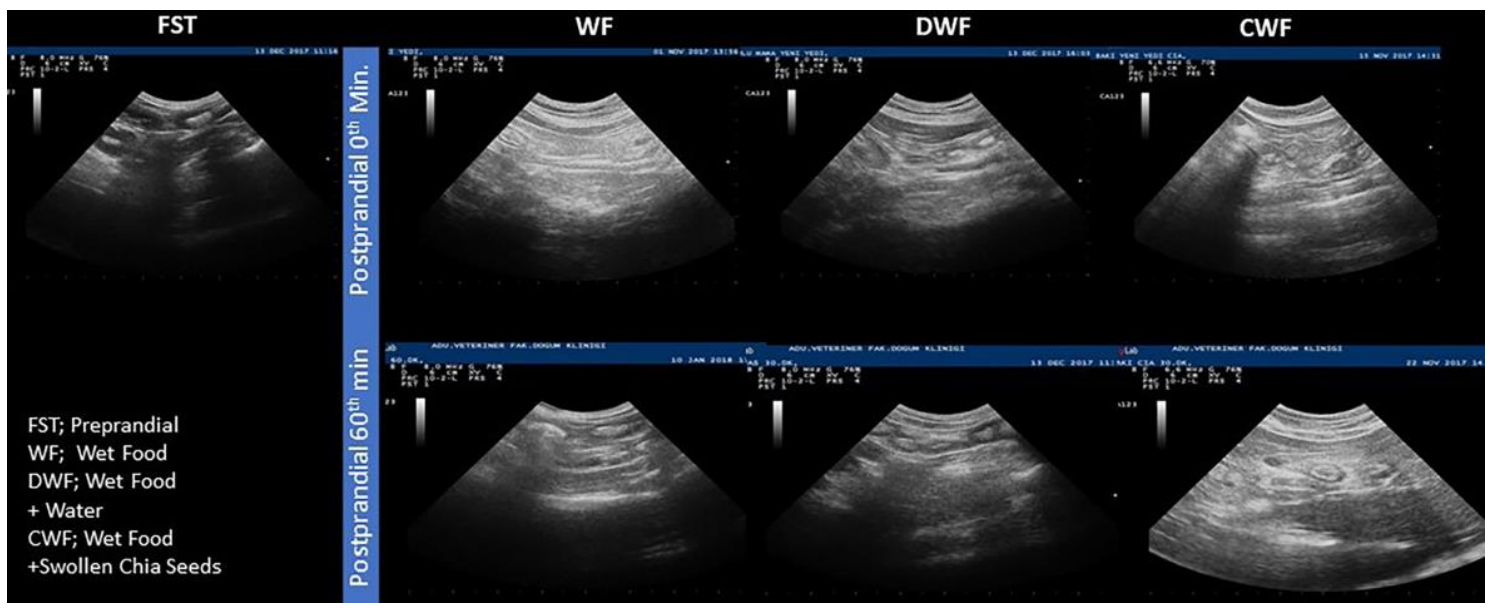

Figure 4. Ultrasonographic images of the ileum at pre- and postprandial 0, 30, 60 minutes

During ultrasonographic imaging of the gastrointestinal tract artifacts such as reverberation, comet-tail and acoustic shadow are frequently seen because of the intraluminal gas (Burk and Feeney, 2003; Penninck, 2008; Agthe, 2009). In our study, although no artifact was observed in the fasted animals' stomach but acoustic shadow artifact was observed due to gas in the intestines. After feeding, reverberation artifacts in the stomach, and reverberation and acoustic shadow artifacts in the intestines were seen mostly.

In the presented study, the stomach when it is empty totally entered to the field of the view together with its rugal folds described as in the literature (Agthe, 2009). Also, the stomach in most animals, could be viewed totally at the all times in CWF and at the 30th and 60th minutes in DWF. There are studies arguing that both the stomach can appear much thicker than normal when the stomach is completely empty (Agthe, 2009), and that gastric enlargement does not cause a significant change in the thickness of the rugalfolds or inter rugal region (Newell et al., 1999). Gastric wall thickness was not measured in this study, but observed that the appearing of the completely empty stomach wall was thicker. In addition, while the stomach walls initially gave a small number of echogenic areas in the all groups, the amount of the echogenicity increased as time passed after the food intake, however, it decreased again in the 60th minute in the CWF.

In cats and dogs, the duodenum descendens and distal ileum can usually be observed in the same area, but other small intestine segments are distributed within the abdomen (Agthe, 2009). The duodenum in cats is close to the midline and has a straight course (Agthe, 2009; Larson and Biller,
2009). In our study, duodenum could be observed in the longitudinal view distal to the stomach in the dorsal recumbency in FST and the nutritional status did not affect the position of the duodenum. Apart from this, according to the type of food taken, the wall echogenicity of the duodenum was affected at different levels at different times. The echogenicity increase that occurs 60 minutes after feeding in dogs is caused by physiological lacteal dilatation (Gaschen et al., 2016). In this study, it was also thought that the echogenicity change over time was caused by mucosal absorption and lacteal dilatation. When evaluated in this way, it can be suggested that Chia seeds addition to the food may shorten both the absorption and transition time.

Carefully scanning the entire middle abdomen is necessary to examine the whole jejunum (Larson and Biller, 2009). The hypoechogenicity observed in the jejunum lumen when fasted turned into hyperechogenicity at the 30th and 60th minutes in WF, however it decreased in the 60th minute in DWF and CWF.

The terminal ileum can be identified in the right dorsal quadrant, close to the ileocecocolic junction, slightly caudal to the costal arch in the right lateral recumbency (Agthe, 2009). In cats, the ileocolic junction is usually seen adjacent to the colic lymph node and just medial to the right kidney and is displayed in a cross section similar to a wagon wheel (Larson and Biller, 2009). In our study, it was generally imaged exactly as described, and the nutritional status did not affect the position of the ileum. Also, it is very likely that the echogenicity changes occurring in the 60-minute examination period are independent of the food type.

Diffuse or multifocal wall thickening and the indistinct wall layers and mesenteric 
lymphadenopathy are seen in cases of gastrointestinal inflammation (Baez et al., 1999; Penninck, 2002; Gaschen et al., 2008; Penninck, 2008). In pathological conditions where intestinal wall infiltration is evaluated, residual ingesta affects mucosal echogenicity (Gaschen et al., 2016). Lines and/or speckles on ultrasonographic images of the intestinal wall that form in pathological conditions such as inflammatory bowel disease can also cause from residual ingesta as artifacts (Sutherland-Smith et al., 2007). Some substance such as oil added to food are reported to reduce the formation of these artifacts (Gaschen et al., 2016, Pollard et al., 2013). Based on this information, food intake containing swollen Chia seeds before ultrasonographic examination will positively affect the echogenicity in ultrasonographic examination was hypothesized in this study.

\section{CONCLUSION}

Swollen Chia seeds and wet cat food mixture can be consumed by cats without any force because it is odorless, tasteless and colorless. The stomach wall can be evaluated easier after the cat consumed it, especially in terms of thickness. In the small intestines, digestive tract content affected the echogenicity and the artifact densitydecreases by Chia seeds addition. It was concluded that ultrasonographic examination after feeding with chia seed added formula can be used as a complementary method following the fasting examination.

\section{ACKNOWLEDGMENTS}

This study is summarized from the first author's master of science thesis

Conflict of Interests: The authors declared that there is no conflict of interests.

Financial Disclosure: The authors declared that this study has received no financial support.

Author's Contributions: ZB and BGK designed the study. BGK performed US examination. BGK and $\mathrm{ZB}$ performed data interpretation. BGK and ZB participated in drafting and revising the manuscript. (BGK: Burcu Gökdemirel Kılıç, ZB: Zeynep Bozkan)

\section{REFERENCES}

Agthe P. Ultrasonography of the gastrointestinal tract and associated organs in dogs and cats. In Pract. 2009; 3:182-188.
Ayerza R, Coates W, Lauria, M. Chia seed (Salvia hispanica 1.) As an omega-3 fatty acid source for broilers: influence on fatty acid composition, cholesterol and fat content of white and dark meats, growth performance, and sensory characteristics. Poult Sci J. 2002; 81:826-837.

Baez JL, Hendrick MJ, Walker LM, Washabau RJ. Radiographic, ultrasonographic, and endoscopic findings in cats with inflammatory bowel disease of the stomach and small intestine: 33 cases (1990-1997). J Am Vet Med Assoc. 1999; 215:349-354.

Burk RL, Feeney DA. Small animal radiology and ultrasound a diagnostic atlas and text. $3^{\text {rd }}$ ed. Philadelphia: WB Saunders; 2003. p.249-470.

Gaschen L, Granger LA, Oubre O, Shannon D, Kearney M, Gaschen F. The effects of food intake and its fat composition on intestinal echogenicity in healthy dogs. Vet Radiol Ultrasound. 2016; 57:546-550.

Gaschen L, Kircher P, Stussi A, et al. Comparison of ultrasonographic findings with clinical activity index (CIBDAI) and diagnosis in dogs with chronic enteropathies. Vet Radiol Ultrasound. 2008; 49:56-64.

Larson MM, Biller DS. Ultrasound of the gastrointestinal tract. Vet Clin North Am Small Anim Pract. 2009; 39:747-759.

Machi J, Takeda J, Sigel B, Kakegawa T. Normal stomach wall and gastric cancer: evaluation with high-resolution operative ultrasound. Radiology. 1986; 159:85-87.

Mattoon JS. Making the most of abdominal ultrasound. In: Southern European Veterinary Conference \& Congreso Nacional AVEPA. 2-4 October, Barcelona, 2009.

Muñoz LA, Cobos A, Diaz O, Aguilera JM. Chia seeds: microstructure, mucilage extraction and hydration. J Food Eng. 2012; 8:216-224.

Newell SM, Graham JP, Roberts GD, Ginn PE, Harrison JM. Sonography of the normal feline gastrointestinal tract. Vet Radiol Ultrasound. 1999; 40:40-43.

Peiretti PG, Meineri G. Effects on growth performance, carcass characteristics, and the fat and meat fatty acid profile of rabbits fed diets with chia (Salvia hispanica 1.) Seed supplements. Meat Sci. 2008; 80:1116-1121.

Penninck DG, Nyland TG, Fisher PE, Kerr LY. Ultrasonography of the normal canine gastrointestinal tract. Vet Radiol Ultrasound. 1989; 3:272-276.

Penninck DG. Gastrointestinal tract. In: Nyland T, Mattoon J, eds. Small Animal Diagnostic Ultrasound. $2^{\text {nd }}$ Edition. Philadelphia: WB Saunders; 2002. p.207-230.

Penninck DG. Gastrointestinal tract. In: Penninck D, D'Anjou MA, eds. Atlas of Small Animal Ultrasonography. Ames (IA): Blackwell Publishing; 2008. p.281-318.

Pollard RE, Johnson EG, Pesavento PA, et al. Effects of corn oil administered orally on conspicuity of ultrasonographic small intestinal lesions in dogs with lymphangiectasia. Vet Radiol Ultrasound. 2013; 54:390-397.

Sutherland-Smith J, Penninck DG, Keating JH, Webster CR. Ultrasonographic intestinal hyperechoic mucosal striations in dogs are associated with lacteal dilation. Vet Radiol Ultrasound. 2007; 48:51-57.

Ullah R, Nadeem M, Khalique A, et al. Nutritional and therapeutic perspectives of chia (salvia hispanica l.): A review. J Food Sci Technol. 2016; 53:1750-1758. 